\title{
AMELIORATION OF PULMONARY ALLOGRAFT INJURY BY ADMINISTERING A SECOND RINSE SOLUTION
}

C. J. Serrick, MSc

A. Jamjoum, MD

A. Reis, MD

A. Giaid, $\mathrm{PhD}$

H. Shennib, MD
Objective: The use of rinse solutions before reperfusing liver allografts has been shown to reduce cell death in rats. Carolina rinse solution (an extracellular solution that contains antioxidants, vasodilators, and other substrates that help prevent ischemia-reperfusion injury) has also been shown to improve liver function clinically in liver transplant recipients. This pilot study evaluates the value of a second pulmonary artery flush before reperfusion of a lung graft. Methods: Six groups of Sprague-Dawley rats $(n=6$ each) were subjected to the following: Group 1 lungs were preserved with modified Euro-Collins solution followed by 24 hours of cold ischemia. Group 2 lungs were treated the same as group 1 but reperfused with blood. Group 3 lungs were preserved in Carolina rinse solution followed by 24 hours of cold ischemia. Group 4 lungs were treated the same as group 3 lungs and then reperfused with blood. Lungs in groups 5 and 6 were preserved with Euro-Collins solution, stored cold for 24 hours, and then rinsed with Euro-Collins or Carolina rinse solution, respectively, before reperfusion with blood. Lungs were subsequently stained with trypan blue solution for 5 minutes. Lung blocks were fixed and embedded in water-soluble methacrylate. Trypan blue-stained nuclei in nonviable endothelial cells and alveolar pneumocytes were counted in 10 different fields. Results: Groups 1 and 3, preserved with Euro-Collins and Carolina rinse solutions for 24 hours but not reperfused with blood, had significantly more viable endothelial cells (groups 1 and 3 vs group 2, $p<0.0001$; group 3 vs group $4, p<0.02$ ) and pneumocytes (group 1 vs groups 2 and 4, group 3 versus group $2, p<0.0001$; group 3 vs group 4 ; $p<0.035$ ) than groups 2 and 4 , which were subsequently reperfused with blood. Groups 5 and 6 , which received a second rinse, also had significantly more viable endothelial cells $(p<0.0005)$ and pneumocytes $(p<0.0001)$ than control groups, which were not rinsed before reperfusion. Conchusions: We conclude that damage to pulmonary allografts resulting from prolonged ischemia is accentuated by reperfusion with blood. We also conclude that preservation with a single flush of Euro-Collins or Carolina rinse solution does not offer adequate protection, whereas a second rinse before reperfusion significantly decreases the number of damaged cells within the allograft. (J Thorac Cardiovasc Surg 1996;112:1010-6)
W hen subjected to prolonged cold ischemia, most solid organs show a cellular injury that primarily involves the vascular endothelium. The lung allograft is invaded with activated neutrophils, lym-

From the Montreal Lung Transplant Program, Montreal, Quebec, Canada.

Received for publication Feb. 13, 1996; revisions requested March 26, 1996; revisions received April 19, 1996; accepted for publication April 22, 1996.

Address for reprints: H. Shennib, MD, Montreal Lung Transplant Program, Montreal General Hospital, 1650 Cedar Ave., Suite L9-120 Montreal, Quebec H3G 1A4, Canada.

Copyright (ㅇ 1996 by Mosby-Year Book, Inc.

$0022-5223 / 96 \$ 5.00+0 \quad \mathbf{1 2 / 1 / 7 4 3 2 8}$ phocytes, and macrophages capable of producing or promoting the release of cytokines and other vasoactive substances, which further accelerates endothelial cell damage. This injury is accentuated after reperfusion. Different types of preservative solutions and different methods of lung preservation have been used to try to the minimize damage after reperfusion of lung allografts. Results, however, have not been convincing, and none of these methods has been widely accepted in clinical practice. The most widespread method of lung preservation is the use of a single pulmonary artery flush with cold, modified Euro-Collins solution (ECS), ${ }^{1}$ an intracellular solution with bloodlike properties originally 
designed for kidney preservation. In contrast to other organs such as the kidney, however, the lung cannot be kept ischemic for more than 12 hours, when ECS is used, without the risk of severe graft dysfunction.

Recently, the use of a second flush with Carolina rinse solution (CRS) before reperfusion has been suggested as a means to improve outcome in liver transplantation recipients. ${ }^{2}$ Currin, Thurman, and LeMasters $^{3}$ reported a decrease in the number of damaged cells in rat liver grafts perfused with a second rinse of CRS when compared with perfusion with University of Wisconsin solution alone. CRS is an extracellular solution with a cocktail of additives such as antioxidants, vasodilators, and different substrates such as glucose and fructose, which may theoretically prevent or counteract the effects of ischemia-reperfusion injury. ${ }^{4}$ The concept of a second rinse is not new, being widely practiced after administration of crystalloid cardioplegic solution in cardiac operations. Among many other potential benefits, the second rinse has been shown to improve buffering and replenish important substrates such as glucose or adenosine triphosphate, which may have become depleted or degraded. ${ }^{5}$ Thus it is likely that this simple method may be advantageously applicable in lung transplantation. The purpose of this study was to determine whether administration of a second rinse of preservative solution before reperfusion could preserve the lungs better than if the lungs were treated with a single flush using the same solutions. In this preliminary study the solutions tested were modified ECS (an intracellular solution) and CRS (an extracellular solution). The effects of this strategy on the integrity and viability of graft pneumocytes and endothelial cells from rat lungs preserved for 24 hours were examined.

\section{Method}

Male Sprague-Dawley rats (250 to $300 \mathrm{gm}$ ) were anesthetized by a mixed intraperitoneal injection of ketamine hydrochloride $(100 \mathrm{mg} / \mathrm{kg})$, xylazine hydrochloride $(6 \mathrm{mg} /$ $\mathrm{kg})$, and atropine $(1 \mathrm{mg} / \mathrm{kg})$ and were orotracheally intubated with a 16-gauge intravenous catheter. Animals were then connected to a pressure-controlled ventilator (Hoek Loos infant ventilator, Hoek Loos Medical Equipment, Amsterdam, The Netherlands) with intermittent positivepressure ventilation (respiratory rate of 60 breaths/min, inspiratory pressure of $1.2 \mathrm{kPa}$, positive end-expiratory pressure of $0.3 \mathrm{kPa}$, and flow rate of $3.5 \mathrm{~L} / \mathrm{min}$ of $100 \%$ humidified, warmed oxygen).

The rats were placed in the supine position and the abdomen was opened by a midline and transverse incision.
The diaphragm was cut from its costal attachment to visualize the thoracic cavity. The right ribs, muscles, and skin were cut near the spine and the anterior chest wall was displaced to the left side. At this time animals received an injection of $300 \mathrm{U}$ of heparin into the inferior vena cava to prevent thrombosis. The right ventricle was punctured with an 18-gauge needle and the lungs were flushed with ice cold preservative solution at a rate of 3 to $4 \mathrm{ml} / \mathrm{min}$ per gram for 2 minutes. The lungs were removed en bloc with the heart and stored at approximately $4^{\circ} \mathrm{C}$ for 24 hours.

All animals were given humane care in compliance with the Animal Care Committee regulations of the Montreal General Hospital and McGill University, as well as with the "Principles of Laboratory Animal Care" formulated by the National Society for Medical Research and the "Guide for the Care and Use of Laboratory Animals" prepared by the National Academy of Sciences and published by the National Institutes of Health (NIH Publication No. 85-23, revised 1985).

Single versus double flush. Six groups $(n=6$ each) were studied. Groups 1 to 4 served as controls and did not receive a second flush.

Group 1. Lungs were preserved with ECS, stored for 24 hours at approximately $4^{\circ} \mathrm{C}$, and then stained with trypan blue stain for 5 minutes (ischemia only with no reperfusion).

Group 2. Lungs were preserved with ECS for 24 hours, like group 1 lungs. Then the lungs were reperfused with warm rat blood at a rate of 3 to $4 \mathrm{ml} / \mathrm{min}$ per gram of tissue for 10 minutes. The lungs were then stained with trypan blue stain (ischemia plus reperfusion).

Group 3. Lungs were preserved with the CRS, stored for 24 hours, and then stained with trypan blue stain for 5 minutes (ischemia only with no reperfision).

Group 4. Lungs were preserved with CRS, stored for 24 hours, and reperfused with blood. The lungs were then stained with trypan blue stain (ischemia plus reperfusion).

In groups 5 and 6 the lungs received a second flush of either ECS or CRS at $4^{\circ} \mathrm{C}$ before reperfusion with blood.

Group 5. Lungs were preserved with ECS, stored for 24 hours at approximately $4^{\circ} \mathrm{C}$, and then rinsed again with ECS at a rate of 3 to $4 \mathrm{ml} / \mathrm{min}$ per gram of tissue for 2 minutes before reperfusion. The lungs were then reperfused with blood and stained with trypan blue stain.

Group 6. Lungs were preserved with ECS, stored for 24 hours as in group 5, and then rinsed with CRS at a rate of 3 to $4 \mathrm{ml} / \mathrm{min}$ per gram of tissue for 2 minutes before reperfusion. The lungs were then reperfused with blood and stained with trypan blue stain.

Histologic assessment of cell viability. Lungs were stained to determine cell viability by the infusion of trypan blue stain, $500 \mu \mathrm{mol} / \mathrm{L}$, through the right ventricle for 5 minutes at a rate of 3 to $4 \mathrm{ml} / \mathrm{min}$ per gram. Lung blocks were then fixed with Poly/LEM fixative, methanol free (Polysciences Inc., Warrington, Pa.). After fixation, the lung tissues were embedded in water-soluble glycol methacrylate, sectioned, and stained with eosin. Trypan blue-stained nuclei of nonviable endothelial cells and pneumocytes were counted in ten fields of power by an individual blinded to the method of preservation. The total number of dead cells was added and recorded. 


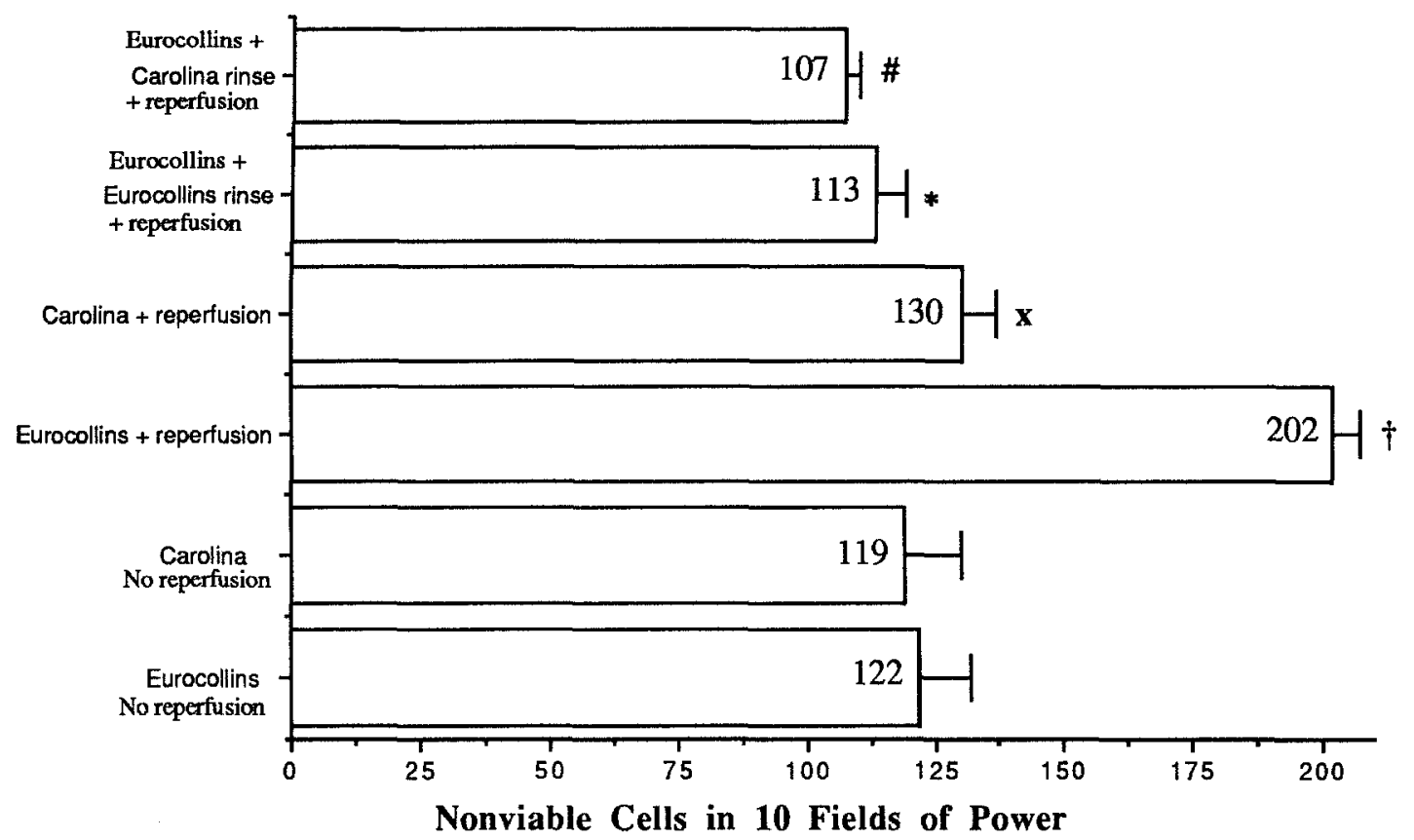

Fig. 1. Endothelial cell viability in rat lungs after 24 hours of cold ischemia. $\# p<0.0001$ versus ECS or CRS + reperfusion, $p=0.008$ versus CRS with no reperfusion, and $p=0.0011$ versus ECS with no reperfusion; ${ }^{*} p=0.0005$ versus CRS + reperfusion, $p<0.0001$ versus ECS + reperfusion, and $p=0.0335$ versus ECS with no reperfusion; $x p=0.0199$ versus CRS with no reperfusion; $\uparrow p<0.0001$ versus ECS or CRS with no reperfusion and CRS + reperfusion.

Statistics. Statistical analysis was determined by means of a one-factor analysis of variance. A $p$ value of 0.05 or less was considered statistically significant. All data are expressed as mean \pm standard error of the mean.

\section{Results}

Assessment of endothelial cell and pneumocyte viability by use of trypan blue staining. Control lungs preserved with ECS or CRS and subjected to 24 hours of ischemia alone (groups 1 and 3) had significantly fewer dead endothelial cells than lungs from their corresponding control groups (groups 2 and 4), which were reperfused with blood (group 1 vs group 2, $p<0.0001$; group 3 vs group $2, p<$ 0.0001 ; group 3 vs group 4: $p<0.02$ ).

Both groups receiving a second flush with ECS or CRS before reperfusion (groups 5 and 6) had significantly fewer dead endothelial cells than control groups 1,2, and 4 (groups 5 and 6 vs groups 2 and 4 , $p<0.0005$; group 5 vs group $1, p<0.035$; group 6 vs group $1, p<0.001$ ). However, no difference was detected between groups 5 and 6 with regard to endothelial cell viability. In lungs rinsed with CRS before reperfusion (group 6), the likelihood of endothelial cell death was significantly lower than in those lungs that had ischemic injury without reperfusion and that were preserved with CRS (group 3) (group 6 vs group 3,p<0.008). This was not the case when lungs were rinsed with ECS (group 5 vs group $3, p=$ not significant; see Fig. 1).

Pneumocyte cell death was significantly less prevalent when ECS was used as the preservative without reperfusion (group 1) than in all other control groups (group 1 vs groups 2,3 , and $4, p<0.0001$ ). Pneumocyte cell death was also significantly less prevalent when CRS was used as the preservative (group 3) than in control groups that were reperfused with blood (group 3 vs groups 2 and $4, p<$ $0.0001)$. Although cell death was more prevalent when CRS was used as the preservative than when ECS was used (group 1 vs group $3, p<0.035$ ), on reperfusion the CRS group (group 4) had significantly more viable pneumocytes than the ECS group (group 2) (group 4 vs group 2, $p<0.0001$ ).

Both groups treated with a second flush (groups 5 and 6) had significantly fewer dead pneumocytes than did the control groups that were not rinsed (groups 1 to 4 ), irrespective of the preservation solution used (groups 5 and 6 vs groups 1 to $4, p<$ 0.002 ). However, there were significantly more dead 


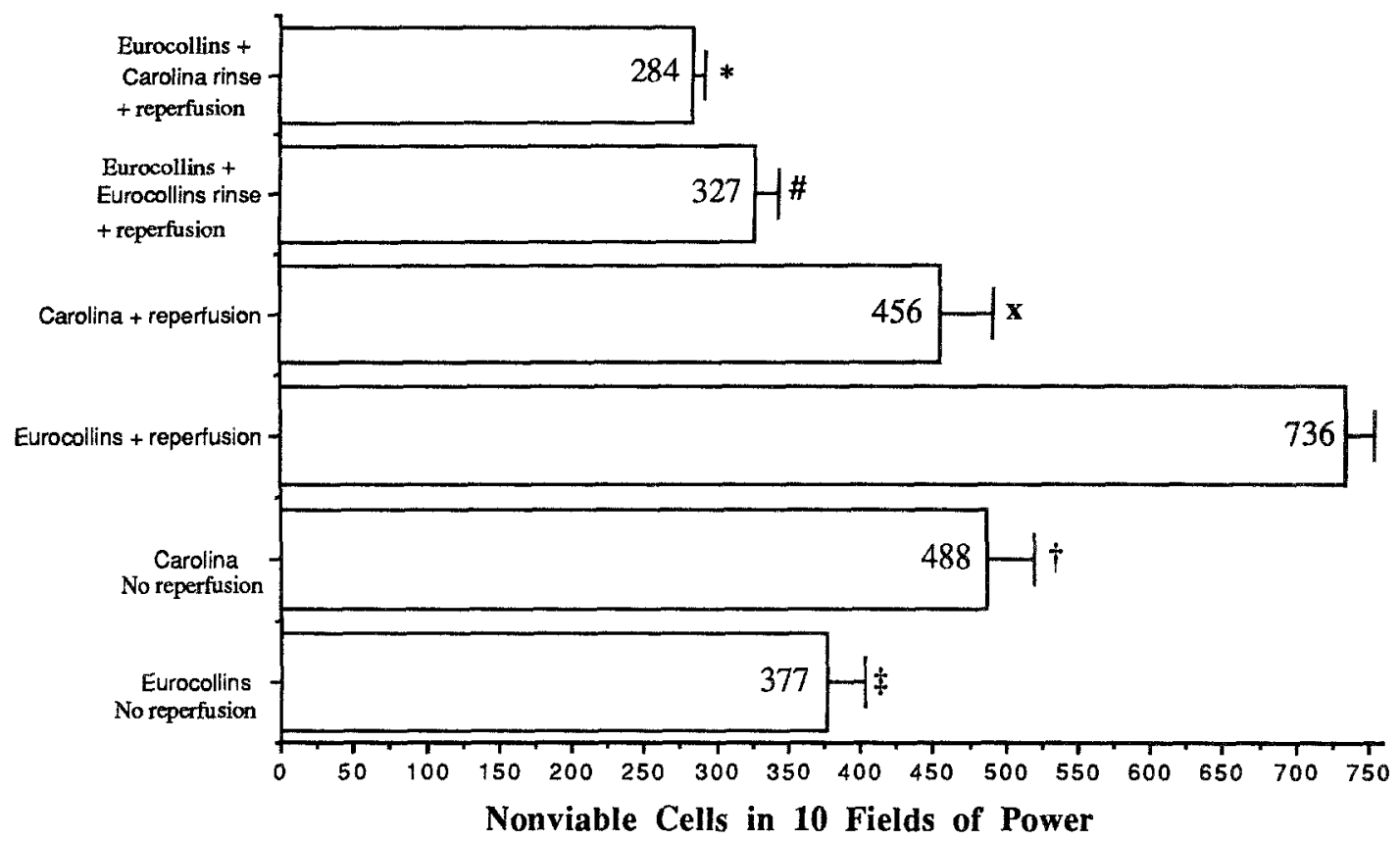

Fig. 2. Pneumocyte viability in rat lungs after 24 hours of cold ischemia. ${ }^{*} p<0.0001$ versus control groups and $p=0.0056$ versus ECS + ECS rinse + reperfusion; \# $p<0.0001$ versus CRS with no reperfusion and $\mathrm{ECS}$ or CRS + reperfusion and $p=0.0017$ versus ECS with no reperfusion; $\dagger_{p}<0.0001$ versus ECS + reperfusion and $p=0.0348$ versus CRS + reperfusion; $x p<0.0001$ versus ECS + reperfusion; $\ddagger p<0.0001$ versus control groups.

pneumocytes when ECS was used as the rinse (group 5) than when CRS was used (group 6) (group 5 vs group $6, p<0.006$; see Fig. 2 ).

\section{Discussion}

During lung transplantation, donor lungs are subjected to an obligatory period of cold and warm ischemia at the time of organ retrieval and implantation. However, it is during the period of reperfusion that most of the organ damage seems to occur through the release of oxygen-derived free radicals and other metabolites. ${ }^{6-8}$ It is for this reason that the major concern early after transplantation has focused on the immediate injury to the lung in what is described as the "reimplantation response." The present study supports this hypothesis, inasmuch as reperfusion resulted in significantly more killing of endothelial cells and pneumocytes than did cold ischemia alone.

During reperfusion injury a complex cascade of various pathogenic mechanisms can cause immediate lung damage. These mechanisms include formation of oxygen-derived free radicals, ${ }^{7,8}$ calcium influx into the cells, ${ }^{9}$ and leukocyte, platelet, ${ }^{10}$ and complement activation. ${ }^{11}$ Likewise, lung function can be improved after transplantation when free radical scavengers such as dimethylthiourea, ${ }^{8}$ superoxide dismutase, and catalase, ${ }^{7}$ are administered before reperfusion. Calcium channel blockers and vasodilators have also been shown to prevent ischemia-reperfusion injury. ${ }^{12}$

CRS (developed at the University of North Carolina) was developed to counteract factors that may cause organ damage after reperfusion. It is an extracellular solution that contains electrolytes similar to plasma $(\mathrm{NaCl}, 115 \mathrm{mmol} / \mathrm{L} ; \mathrm{KCl}, 5 \mathrm{mmol} / \mathrm{L}$; $\mathrm{CaCl}_{2}, 1.3 \mathrm{mmol} / \mathrm{L} ; \mathrm{KH}_{2} \mathrm{PO}_{4}, 1 \mathrm{mmol} / \mathrm{L}$; and $\left.\mathrm{MgSO}_{4}, 1.2 \mathrm{mmol} / \mathrm{L}\right) .{ }^{1}$ Other constituents include antioxidants such as allopurinol, desferrioxamine, and glutathione; vasodilators such as nicardipine and adenosine; hydroxylethyl starch to protect against edema; and fructose, glucose, and insulin to help regenerate adenosine triphosphate stores. ${ }^{3}$

In this study, we have shown that a second pulmonary artery flush before reperfusion, regardless of the type of rinse solution used, is beneficial to the lung graft by preventing lethal injury to endothelial cells and pneumocytes. This may be attributed to the flushing out of metabolites such as 
oxygen-derived free radicals, activated cells, and components of complement that can damage the lung parenchyma. Although a second flush with either ECS or CRS did protect the lung, CRS provided greater protection than did ECS. Pneumocyte and endothelial cell death was significantly less prevalent in lungs preserved with CRS. This difference can be attributed to the properties of the solution itself. Direct observation of the hepatic microcirculation by means of in vivo microscopy has shown that CRS had a beneficial effect on microcirculatory manifestations of ischemia-reperfusion injury in rat liver grafts. Both microcirculatory perfusion failure and white blood cell accumulation and adherence were reduced after grafts were rinsed with CRS. ${ }^{13}$ A substantial decrease in vascular resistance is apparent after perfusion with CRS. The main cause for this decreased resistance is believed to be protection of the endothelial cells of the microvasculature. Thus Sato and colleagues ${ }^{14}$ have demonstrated that sinusoidal endothelial cells of the liver were better protected by CRS than by other solutions used as a rinse after 24 hours of storage with University of Wisconsin solution. Also, several components of CRS have been shown previously to protect against lethal injury to cells depleted of adenosine triphosphate, including fructose, ${ }^{15}$ acidic $\mathrm{pH},{ }^{16}$ the antioxidants desferrioxamine and allopurinol, ${ }^{17,18}$ and dihydropyridine-type calcium channel blockers. ${ }^{3}$

On the other hand, ECS is an intracellular solution with a low sodium, high potassium concentration and has little protective effect against reperfusion injury other than providing cells with a homeostatic environment during the storage or ischemic stage. It causes pulmonary vasoconstriction ${ }^{19}$ in lung allografts and has been shown by many authors to produce poorer airway pressures, lung compliance, and pulmonary vascular resistance after long ischemic periods than do other lung storage solutions. $^{20-25}$

The type of preservation solution to be used during lung procurement has been the subject of much debate. In fact, controversy still exists as to whether an intracellular or extracellular solution is better. Experimentally and to a certain extent clinically, both intracellular and extracellular products have yielded favorable results. The current method used by most centers practicing clinical lung transplantation is the standard pulmonary artery flush with modified ECS, ${ }^{2}$ an intracellular solution modified with bicarbonate and magnesium. However, other intracellular solutions such as the University of Wisconsin solution have been shown experimentally in dogs to be superior to ECS as a preservative for lung transplantation, ${ }^{26,27}$ with extended preservations times of up to 24 hours. On the other hand, extracellular solutions such as low-potassium dextran have provided significantly better lung preservation than did ECS after 12 and 18 hours of ischemia. ${ }^{27}$ In the present study, endothelial cell death after reperfusion with blood was significantly more prevalent in lungs flushed with modified ECS after 24 hours of ischemia than in lungs that were ischemic but not reperfused. However, rinsing the graft with an extracellular solution before reperfusion resulted in significantly less killing of endothelial cells. The CRS (extracellular) protected pneumocytes better before the reperfusion stage than did ECS (intracellular) as determined by the presence of significantly more viable pneumocytes after reperfusion with CRS.

This observation supports a potentially beneficial role of extracellular rather than an intracellular solutions in lung transplantation. The rationale for using a high-potassium solution was thought to be the abolishment of the transmembrane potassium ion gradient, which might decrease cell swelling directly and would limit potassium ion leakage from preserved cells. ${ }^{28}$ However, intracellular solutions have been considered to induce pulmonary vasoconstriction during flushing, causing heterogeneous distribution of the preservative solution and incomplete protection through cooling. ${ }^{29}$ This may also result in an increase in passenger donor blood cells in the pulmonary vasculature. A high potassium concentration may also acutely raise pulmonary artery flush pressures, which may disrupt endothelial cell integrity and contribute to pulmonary edema both during pulmonary artery flushing and during postischemic reperfusion. ${ }^{30}$ In fact, endothelial cells were better preserved with CRS than with ECS, regardless of whether CRS was used as a preservative or rinse solution. This distinction did not obtain for pneumocytes, inasmuch as ECS used as a preservative and rinse solution also helped to prevent pneumocyte death. However, significantly more pneumocytes died after reperfusion when ECS was used as both a preservative and a second flush than when CRS was used as the second rinse. The ECS rinse decreased pneumocyte death, but not to the same extent noted when CRS was used before reperfusion. This observation agrees with evidence from rat pneumocyte cell culture models, which 
suggest that incubation in high-potassium intracellular solutions may be more injurious than a variety of low-potassium preservation solutions under cell culture conditions. ${ }^{31,32}$

Despite the suggested benefits of using drugs that act like oxygen-derived free radical scavengers or vasodilators, the most common preservation solution currently used is ECS. In this pilot study using a simple in vitro model of ischemia-reperfusion injury, we have shown the superiority of an extracellular solution as a preservative and also have demonstrated a benefit of using a second rinse by reducing the number of injured endothelial cells and pneumocytes after 24 hours of ischemia. It is possible that the CRS minimizes reperfusion injury by maintaining cell membrane integrity. Further studies are required to evaluate the effect of a second rinse on pulmonary graft function in vivo. We are currently using a canine left lung allotransplantation model observed for 6 hours of reperfusion to assess the adequacy of a second rinse solution with emphasis on functional outcome.

Many thanks to Dr. John Lamasters from the University of North Carolina for the generous supply of CRS.

\section{REFERENCES}

1. Sanchez-Urdazpal L, Gores GL, Lemasters JJ, Thunnan RG, Steers JL, Wahlstrom $\mathrm{HE}$, et al. Carolina rinse solution decreases liver injury during clinical liver transplantation. Transplant Proc 1993;25(1pt2):1574-5.

2. Novick RJ, Menkins AH, Mekenzie FN. New trends in lung preservation: a collective review. J Heart Lung Transplant 1992;11:377-92.

3. Currin RT, Thurman RG, LeMasters JJ. Carolina rinse solution protects adenosine triphosphate-depleted hepatocytes against tethal cell injury. Transplant Proc 1993;23(lpt1): 645-7.

4. Currin RT, Toole JG, Thurman RG, Lemasters J. Evidence that Carolina rinse solution protects sinusoidal endothelial cells against reperfusion injury after cold ischemic storage of rat liver. Transplantation 1990;50:1076-8.

5. Principles of formulation and administration. In: Hearse DJ, Braimbridge MV, Jynge $P$. editors. Protection of the ischemic myocardium: cardioplegia. New York: Raven Press, 1981: 313.

6. Koyame I, Toung TJ, Rogers MC, Gutner GH, Traystman RJ. $\mathrm{O}_{2}$ radicals mediate reperfusion lung injury in ischemic $\mathrm{O}_{2}$ ventilated canine pulmonary lobe. J Appl Physiol 1987;63: 111-5.

7. Paull DE, Keagy BA, Kron EJ, Wilcox BR. Reperfusion injury in lung preserved for 24 hours. Ann Thorac Surg 1989;47:187-92.

8. Detterbeck FC, Keagy BA, Kron EJ, Wilcox BR. Oxygen free radical scavengers decrease reperfusion injury in lung transplantation. Ann Thorac Surg 1990;50:204-10.

9. Borgers M, Thome F, Reempts JV, Verheyen F. The role of calcium in cellular dysfunction. Am J Emerg Med 1983;2:15461.

10. Qayumi AK, Jamieson WRE, Poostizadeh A. Effect of platelet activating factor antagonist CV-3988 in preservation of heart and lung for transplantation. Ann Thorac. Surg 1991;52:1026-32.

11. Ljungman AG, Grum CM, Deeb GM, Boiling SF, Morganroth ML. Inhibition of cyclooxygenase metabolite production attenuates ischemia-reperfusion lung injury. Am Rev Respir Dis 1991;143:610-7.

12. Hachida M, Morton DL. The protection of ischemic lung with vempamil and hydralazine. $\mathbf{J}$ Thorac Cardiovasc Surg 1988;95:178-83.

13. Palma P, Post S, Rentsch M, Gonzalez AP, Menger MD, Messmer K. effects of Carolina rinse on hepatic microcirculation and leukocyte-endothelium interaction in rat liver transplantation. Transplant Proc 1993;25:2536-7.

14. Sato E, Negita M, Hachisuka T, Ohtsuka S, Yokoyama I, Takagi H. Effect of Carolina rinse solution in cultured rat sinusoidal endothelial cells. Transplant Proc 1993;25:3234.

15. Anundi I, King J, Owen DA, Schneider H, Lemasters J, Thurman RG. Fructose prevents hypoxic cell death in liver. Am J Physiol 1987;253:G390.

16. Gores GJ, Nieminen AL, Fleishman KE, Dawson TL, Herman B, LeMasters JJ. Extracellular acidosis delays onset of cell death in ATP depleted hepatocytes. Am J Physiol 1988;235:C315.

17. Bellomo G, Orrencris S. Altered thiol and calcium homeostatsis in oxidative hepatocellular injury. Hepatology 1985;5:876.

18. Ganote CH, Kaltenbach JP. Oxygen-induce enzyme release: early events and a proposed mechanism. J Mol Cell Cardiol 1979;11:389.

19. Unruh, Oppensack M, Openheimer L. Vascular properties of canine lungs perfused with Euro-Collins solution and prostacyclin. Ann Thorac Surg 1990;49:292-8.

20. Keshavjee SH, Yamazaki F, Cardoso PF, McRitchie DI, Patterson GA, Cooper JD. A method for safe 12-hour pulmonary preservation. J Thorac Cardiovasc Surg 1989;98: 529-34.

21. Yamazaki K, Yokomise H, Keshavjee SH, et al. The superiority of an extracellular fluid solution over Euro-Collins' solution for pulmonary preservation. Transplantation 1990; 49:690-4.

22. Semik M, Moller F, Lange V, Bernhadr A, Toomes H. Comparison of Euro-Collins and UW solutions for lung preservation using the parabiotic rat perfusion model. Transplant Proc 1990;22:2235-6.

23. Kawahara K, Ikari H, Hisano H, Takahashi T, Honshou S, Ayabe $H$, et al. Twenty four hour canine lung preservation using UW solution. Transplantation 1991;51:584-7.

24. Naka $Y$, Shirakura $R$, Matsuda $H$, Nakata S, Kawaguchi A, Fukushima N, et al. Canine heart-lung transplantation after 24-hour hypothermic preservation. Eur J Cardiothorac Surg 1990;4:499-503.

25. Naka Y, Shirakura R, Matsuda H, Nakata S, Fukushima N, Nakano $S$, et al. Canine heart-lung transplantation after 24-hour hypothermic preservation with Belzer-UW solution. J Heart Lung Transplant 1991;10:296-303.

26. Kawahara K, Ikarri H, Hisano H, Takahashi T, Honshou S, Ayabe $\mathrm{H}$, et al. Twenty four hour canine lung preservation using UW solution. Transplantation 1991;51:584-7. 
27. Keshavjee SH, Yamazaki F, Cardoso PF, McRitchie DI, Patterson GA, Cooper JD. A method for safe 12 hour pulmonary preservation. J Thorac Cardiovasc Surg 1989;98:529-34.

28. Collins GM, Bravo-Shugarman M, Teraski PI. Kidney preservation for transplantation: initial perfusion and 30 hours' ice storage. Lancet 1969;2:1219.

29. Cooper JD. Current status of lung transplantation. Trans Proc 1991;23:2107-14.

30. Puskas JD, Cardosu PFG, Mayer E, Shi S, Slutsky AS, Patterson GA. Equivalent 18 hour lung preservation by pulmonary flush with low potassium dextran or Euro-Collins solution after prostaglandin $\mathrm{E}_{1}$ infusion. $J$ Thorac Cardiovasc Surg 1992;104:83-9.

31. Puskas J, Maccherini M, Keshavjeee S, Slutsky AS, Patterson GA, Edelson JD. Low potassium dextran is superior as a preservative solution in type II pneumocyte cell culture model. Am Rev Respir Dis 1990;141:A406.

32. Hachida M, Hoon DS, Morton DC. A comparison of solutions for lung preservation using pulmonary alveolar type II cell viability. Ann Thorac Surg 1988;45:643-6. 